East Tennessee State University

Digital Commons @ East Tennessee State University

\title{
CTRP3 and Alcoholic Liver Disease in Female Mice
}

Callie Root

Follow this and additional works at: https://dc.etsu.edu/honors

Part of the Animal Experimentation and Research Commons, Biological Factors Commons, Digestive System Commons, Digestive System Diseases Commons, Laboratory and Basic Science Research Commons, Other Physiology Commons, and the Therapeutics Commons

\section{Recommended Citation}

Root, Callie, "CTRP3 and Alcoholic Liver Disease in Female Mice" (2020). Undergraduate Honors Theses. Paper 541. https://dc.etsu.edu/honors/541

This Honors Thesis - Withheld is brought to you for free and open access by the Student Works at Digital Commons @ East Tennessee State University. It has been accepted for inclusion in Undergraduate Honors Theses by an authorized administrator of Digital Commons @ East Tennessee State University. For more information, please contact digilib@etsu.edu. 


\title{
CTRP3 and Alcoholic Liver Disease in Female Mice
}

\author{
Callie Root \\ April 2020
}

An Undergraduate Thesis Submitted in Partial Fulfilment of the requirements for the

Honors Scholars Program Honors College

and the

College of Public Health

East Tennessee State University

Callie Poot

Callie Root

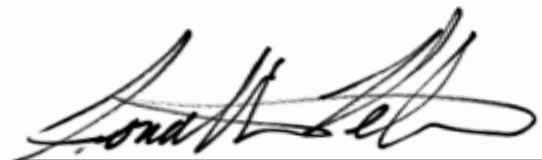

$4 / 21 / 20$

Dr. Jonathan M. Peterson, Thesis Mentor Date

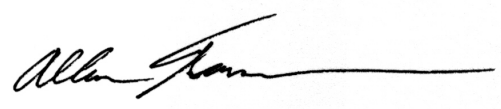

$4 / 21 / 20$

Dr. Allan Forsman, Reader

Date 


\section{Table of Contents}

$\begin{array}{ll}\text { ABSTRACT } & 3\end{array}$

$\begin{array}{ll}\text { ACKNOWLEDGEMENTS } & 4\end{array}$

INTRODUCTION / LITERATURE REVIEW 5

$\begin{array}{ll}\text { METHODS } & 13\end{array}$

$\begin{array}{ll}\text { RESULTS } & 17\end{array}$

$\begin{array}{ll}\text { DISCUSSION } & 21\end{array}$

$\begin{array}{ll}\text { CONCLUSION } & 24\end{array}$

$\begin{array}{ll}\text { REFERENCES } & 25\end{array}$ 


\begin{abstract}
C1q TNF Related Protein 3 (CTRP3), is a cytokine that is primarily secreted from adipose tissue, which classifies it as an adipokine. Our previous research has shown that CTRP3 prevents alcoholic fatty liver disease (ALD) in male mice. However, even when accounting for confounding factors such as absolute and relative alcohol intake, females are more sensitive to the effects of consumption compared to male mice. Therefore, the goal of this project was to determine whether CTRP3 prevented ALD in female mice. Methods: Female wild type (WT) and female CTRP3 transgenic over expressing (Tg) mice were fed an ethanol containing liquid diet (5\% v/v) for 6 weeks. Daily weight and food intake measurements were taken and external heat-pads were placed under a portion of the cage to facilitate thermoregulation. Hepatic steatosis was determined by total triglyceride quantification and lipid droplet quantitation in liver sections. Data were analyzed by repeated measures ANOVA, t-test, or Log-rank (Mantel-Cox) test as appropriate. Results: There was no difference between WT and Tg mice in food intake or body weight. There was no difference in survival between $\mathrm{WT}$ and $\mathrm{Tg}$ mice, however, $\mathrm{Tg}$ mice trended towards a reduced rate of survival compared with WT mice ( $78 \%$ in WT versus $44 \%$ in $\mathrm{Tg}, \mathrm{p}=0.13)$. Stereological analysis indicated no difference in the percent of lipid liver volume between the two groups (WT 7.2 \pm 3.6 vs Tg 5.1 $\pm 4.1 \%$ ). This finding was consistent with no difference in total hepatic triglyceride accumulation observed between WT and Tg mice $(12.7 \pm 4.4 \mathrm{vs} .13 .1 \pm 6.8 \mathrm{mg}$ triglycerides/gram liver protein). Conclusion: Combined these data indicate that unlike previous studies with male mice, CTRP3 is not protective against alcohol-induced hepatic steatosis in female mice. Combined, these data indicate that the adipokines such as CTRP3 contribute to physiology in a sex-specific manner.
\end{abstract}




\section{ACKNOWLEDGEMENTS}

I would first like to thank East Tennessee State University for the personal growth and educational opportunities it has granted me during my four-year undergraduate degree. Likewise, I would like to thank Dr. Karen Kornweibel and the University Honors Scholar program for believing in me and giving me the opportunity and the motivation to complete an undergraduate thesis. I would like to greatly thank Dr. Peterson for the opportunity to work in the lab and for the continual guidance, support and confidence he has instilled in me in these past two years. I would like to thank my fellow lab members as I truly could not do this without them and their help. I would also like to thank Dr. Forsman for agreeing to be a reader and providing guidance in this journey. Lastly, I would like to thank my family and friends, especially my parents, for constantly supporting me and believing in me even when I did not. 


\section{INTRODUCTION / LITERATURE REVIEW}

The National Institute of Alcohol Abuse and Alcoholism defines moderate drinking levels as up to one drink a day for women and two drinks a day for men. In turn, it defines binge drinking as a pattern of alcohol consumption that brings the blood alcohol level to $.08 \mathrm{~g} / \mathrm{dL}$, with heavy alcohol use defined as repeating this pattern five of more days in a month. The combination of both of these tendencies can lead to an alcohol use disorder and severe bodily effects such as Alcoholic Fatty Liver disease (ALD) (Drinking Levels Defined, n.d.) ALD can be defined as a multi-stage process characterized by the presence of hepatic lesions on the liver which can be identified as steatosis, hepatitis, and fibrosis/cirrhosis (in order of severity) (Osna, Donohue, \& Kharbanda, 2017). Hepatic steatosis (fatty liver) is a point at which hepatic lesions accumulate on the liver and account for $5 \%$ of total liver weight with no signs yet of inflammation. In turn, hepatitis ensues when inflammation is present, and cirrhosis is a point at which fibrosis is detected (Sakhuja, 2014). Importantly, ALD has a large cultural impact that can have detrimental effects to one's livelihood and lifestyle. Currently, there is no modern diagnostic tool to test the susceptibility to the development of ALD, and the pathogenies is still currently not completely understood (Gao \& Bataller, 2011). More importantly, what makes this research relevant is the fact that there is currently no FDA-approved treatment on the market. As of now, the most effective treatment is seen as the cessation of drinking, and liver transplant if the disease progresses to a state as to where liver function is inadequate to support bodily function (Osna, Donohue, \& Kharbanda, 2017). Another reason for interest in the topic is due to the fact that it has already been shown that there are noticeable differences between severity and presence of ALD between male and females. For example, women are more susceptible to alcohol-related liver damage than men, and women achieve higher blood alcohol content levels 
as compared to men after drinking equivalent amounts of alcohol (Mumenthaler, Taylor, O'Hara, \& Yesavage, 1999). In regard to mice specifically, it has been found that females have a higher mortality rate in response to chronic alcohol fed male mice (Trogen, et al., 2018). Because of this, we are interested to learn about the different mechanisms and factors that play into presence of and protection from ALD, and how we could possibly use these to make strides in decreasing the severity of the effects, or ultimately formulating a cure.

The liver is a vital organ for bodily function as one of its main goals is to act as a filter for blood that is going through the digestive tract. Likewise, it produces bile and other phospholipids (Knell, 1980). Although early stages of liver damage are asymptomatic, as the disease progresses ALD can not only affect the liver itself, but begin to show negative effects in other bodily functions. For the sake of this particular research we are specifically interested in the effect of excess alcohol consumption and ALD on adipose tissue. Adipose tissue plays an important role in storing excess fat in adipocytes while also acting as an active endocrine organ that can excrete both hormones and cytokines (Lundbom, 2017). Adipose tissue can be affected by alcohol in a multitude of different ways including both the mass of adipokines and adipokine secretion (Kema, Mojerla, Khan, \& Mandal, 2015). Adipokines are secreted from adipose tissue and can have various effects throughout the body. These can be seen in instances such as their implications in insulin sensitivity, alterations of metabolic regulation and other homeostatic imbalances (Deng \& Scherer, 2010). Adipokines come in many different specified forms that have been named and identified in accordance to their function and roles within body systems and placed into groups such as hormones, growth factors, angiogenic factors and cytokines (Pereira \& Alvarez-Leite, 2013). For our research, we were particularly interested in the effect of alcohol on members of the C1q tumor necrosis factor (TNF)- related protein (the CTRP) 
family. These adipokines present themselves to have a wide range of bodily impacts or areas functions such as metabolism, inflammation, and survival signaling in multiple tissue types ( $\mathrm{Li}$, Wright, \& Peterson, 2017).

C1q/TNF-Related Protein 3 (CTRP3) is a particularly significant secreted adipokine that has many different implications throughout the body. As it has been the center of many past studies, it has been found to be associated with factors such as triglyceride levels, glucose regulation and insulin sensitivity (Trogen, Alamian \& Peterson, 2019). Further, it has been shown to increase adipokine secretion, attenuate inflammatory signaling, promote proliferation, increase cellular differentiation and increase hepatic lipid oxidation (Li, Wright, \& Peterson, 2017). Another interesting characteristic of CTRP 3 is its tendency to demonstrate sex specific regulation and function (DeGroat A. R., et al., 2018). CTRP3 has been tied to similar liver dysfunction as seen in Non Alcoholic Fatty Liver disease (NAFLD) as it has shown to be protective against hepatic steatosis (Peterson, Seldin, \& Wong, 2013). Studies looking at Nonalcoholic fatty liver disease show that CTRP3 levels are reduced in human patients who have been diagnosed with NAFLD, signaling the possibility of CTRP3 playing a protective role in NAFLD prevention (Peterson, Seldin, \& Wong, 2013). Because of this correlation between CTRP3 and its attenuation of fatty liver disease, experimentation has begun to see if a correlation can also be found with the development and progression of ALD (DeGroat A. R., et al., 2018).

In regard to CTRP3 and ALD specifically, there is relevant research that demonstrates the basis of interest between the correlation of the two, and the differences in severity and presence of ALD based on factors such as sex. Primarily, CTRP3 has been shown to act protectively against long term alcohol-induced lipid accumulation. This leads to the hypothesis that, combined with cessation of alcohol consumption, CTRP3 can be beneficial in treating ALD 
(DeGroat A. R., et al., 2018). Because of this initial question, a publication entitled "The Sex specific effect of alcohol consumption on circulating levels of CTRP3" (DeGroat A. R., et al., 2018) provides an extensive look at the effect of alcohol consumption on circulating adipokine levels (with CTRP3 being the main focus). Wildtype C57B16 mice were exposed to two different ethanol containing diets. One diet mimicked both chronic combined with binge feeding (NIAAA model), and the other a chronic with no binging component (chronic model) (Bertola, Mathews, Ki, Wang, \& Gao, 2013). Utilizing these feeding models, the sex differentiating effects of CTRP3 can be exemplified by analyzing the adipokine levels and mortality rates. Likewise, it displayed that the effects of the chosen feeding models and adipokines vary as well. This research provided us with data that there is indeed a difference between adipokine expression in male and female mice. Mortality rate differences showed that females suffered approximately $50 \%$ mortality during the 6-week alcohol feeding study, while in males, there was a no difference in mortality between control and ethanol fed mice (DeGroat A. R., et al., 2018). This shows the sex specific effects of an alcohol containing diet. Further, circulating CTRP3 levels in females decreased by $\sim 75 \%$ in the NIAA model and $\sim 50 \%$ in the chronic model while no changes were observed in males (DeGroat A. R., et al., 2018). Because CTRP3 levels have been shown to be selectively reduced in ethanol $(\mathrm{ETOH})$ fed females, this could serve as a possible mechanism for the increased susceptibility of females to alcoholic cirrhosis (DeGroat A. , et al., 2018).

While this data was focused mainly on CTRP3, other adipokines were examined as well. Circulating adiponectin, determined by immunoblot, show that levels increase in both the NIAAA and chronic feeding models in females, and increased in males only in the chronic model (DeGroat A. R., et al., 2018). Adiponectin is a peptide that is secreted by adipocytes and 
has been shown to play a role in insulin resistance, type 2 diabetes, and cardiovascular disease (Acharie \& Jain, 2017). Adiponectin is related to ALD in particular because increased adiponectin levels have previously been correlated to patients with cirrhosis (Gamberi, Maherinie, Modesti, \& Fiashi, 2018). With adiponectin it is important to note the effect of chronic alcohol abuse demonstrating a positive association between circulating adiponectin levels and the severity of liver damage (Tang, et al., 2012). It is interesting to note that even though both males and females show an increase in adiponectin concentration, females displayed a $\sim 200 \%$ increase in circulating levels in the NIAAA model while males had no noticeable changes in circulating levels. In regard to the chronic diet, adiponectin levels doubled in both males and females (DeGroat A. R., et al., 2018). This data demonstrated the differing effects that both the feeding model type and sex can have on adiponectin levels. This data displayed an increase in both male and female mice that showed activating adiponectin mediated signaling pathways is not a particularly successful strategy for prevention/treatment of ALD due to levels already increasing with chronic ethanol exposure (DeGroat A. R., et al., 2018). The increase of adiponectin levels found in the NIAAA model female ETHOH fed mice may be used to account for the historic increased severity of ALD in females. These findings, along with many other notable discoveries that were made in the manuscript exemplify the sex differences in actions of adipokines and ALD. This can lead to further studies to examine the relationship between adipokines and ALD more in depth as well as CTRP3 as a potential mechanism for the increased susceptibility of females to ALD.

With this knowledge that there is a correlation between CTRP3 and ALD, further study was warranted to see if overexpression of CTRP3 can prevent ALD (Trogen, et al., 2018). In a publication entitled, "Transgenic overexpression of CTRP3 prevents alcohol-induced hepatic 
triglyceride accumulation" (Trogen, et al., 2018) the exploration of this possible intervention method is made through the use of WT and Tg+ CTRP 3 overexpressed mice. Similarly, the NIAAA (used to mimic the hepatic steatosis and liver injury and inflammation that occur in many alcoholic hepatitis patients) and chronic fed model were employed identically to the previous study (Bertola, Mathews, Ki, Wang, \& Gao, 2013). The primary findings showed that the overexpression of CTRP3 reduced triglyceride accumulation in the 6-week ethanol feeding, but not the NIAAA model (Trogen, et al., 2018) Because of the previously formulated thesis of CTRP3 attenuating alcohol-induced hepatic lipid accumulation, the study expected to see a protection of livers in the Tg-ETOH mice. But, there was no statistically significant protection observed in the NIAAA model (Trogen, et al., 2018).

However, transgenic overexpression of CTRP3 completely abolished ethanol-induced hepatic steatosis in response to chronic ethanol feeding, including reversing the ethanol-induced changes to the hepatic fatty acid profile (Trogen, et al., 2018). These finding indicate that increased circulating levels of CTRP3 can act protectively over time in male mice (Trogen, et al., 2018). The discrepancy between the two feeding models are thought to be due to the fact that liver triglyceride accumulation (the variable concentrations that are being studied) in the NIAAA model may be secondary to hepatic inflammation, and that CTRP3 levels were not sufficient to improve the initial inflammation and triglyceride accumulation (Trogen, et al., 2018). The resulting data also indicated that CTRP3 attenuated the effects of alcohol consumption on fatty acid oxidation or fatty acid elongation and desaturation in male mice (Trogen, et al., 2018). To further explore this correlation between CTRP3 and attenuation of hepatic steatosis, the study examined the phosphorylation status (activity levels) of many key enzymes in the regulation of lipid metabolism. Notably, there was an observed decrease in ERK1/2 and AMPK 
phosphorylation in the Tg-ETOH group compared to that of the WT-ETOH (Trogen, et al., 2018). Factors such as Akt, ACC, DGAT2 and SREBP1 showed no change in phosphorylation indicating that CTRP3 did not activate these enzymes (Trogen, et al., 2018). This is relevant due to the previously indicated implications of treatments focused on the blocking of ERK1/2 are associated with alcohol-induced hepatic damage (Li, C. et al., 2017). But this warrants further study as there are many contradictory beliefs in regard to the role of phosphorylated ERK1/2 in relation to possibly increasing sensitivity and first-hit and second-hit ALD (Trogen, et al., 2018).

In addition, AMPK levels were seen to be elevated in WT-ETOH male mice compared to that of the Tg-ETOH male mice, with no difference in the phosphorylation status of ACC (Trogen, et al., 2018). Importantly, even though the mechanism of the action of AMPK is still fully not known, the elevated AMPK levels supports our hypothesis that the overexpression of CTRP3 attenuates the rise in AMPK phosphorylation which leads to lower total triglyceride accumulation. Once again further studies are warranted to further understand the mechanism between ACC phosphorylation and AMPk stimulation and the effect this truly has on ALD (Trogen, et al., 2018). The effects of CTRP3 in isolated hepatocytes was examined to see if CTRP3 over expression stimulates fatty acid oxidation (Trogen, et al., 2018). This is relevant due to the role of NADH promoting steatosis by stimulating the synthesis of fatty acids, in turn opposing their oxidation (Lieber, 2004). The collected data indicated that CTRP3 does not increase basal metabolism and CTRP3 (with added palmitic acid) increased oxygen consumption. Together, this data displays that CTRP3 stimulates lipid metabolism in the liver (Trogen, et al., 2018).

An important limitation of the study above is that due to budgetary restrictions, only male mice were used in the study (Trogen, et al., 2018). In fact, this is a trend often replicated across 
many fields of scientific research in regard to both mice and humans. Historically, fewer women are included in research, and results are often not reported by sex (Mazure \& Jones, 2015). This is done because of the idea that female animal subjects will increase cost and study variability. But in reality, this variability is necessary due to the varying cross-sex differences evident in medicine and disease (Fields, 2014). Due to the previous research displaying that females are historically more susceptible to ALD, further studies should place focus on whether or not female mice under similar conditions would express the same results (Bellentani, et al., 1997). This in addition to the previously established notable sex differentiation upon cytokines, mortality, and ETOH feeding models, provides a reason to further explore the effect of cytokines on females (DeGroat A. R., et al., 2018). This is the basis for our hypothesis and reasoning for replicating the previously mentioned "Transgenic overexpression of CTRP3 prevents alcoholinduced hepatic triglyceride accumulation" study to include a Tg+ CTRP3 overexpressing female mice group compared to that of a WT-ETOH fed female group. We hypothesize that the data will display that CTRP3 acts protectively in females against ALD, but to a lesser extent to account for the increased severity and progression of ALD in females versus males. 


\section{METHODS}

\section{Animal Model}

The CTRP3 transgenic overexpression (Tg) mouse strain was developed on the C5BI/6J

background with the carboxy-terminal FLAG epitope (DYKDDDDK)-tagged CTRP3 expression driven by the CMV early enhance/chicken $\beta$-actin (CAG) promoter as described in (Peterson, Seldin, \& Wong, 2013). Female mice were housed in polycarbonate cages on a 12-hour light/dark photocycle with ab libitum access to food and water, except as specified. Controls for this experiment were Wildtype (WT) littermates. Mice were inspected and weighed daily. At the time points indicated, animals were anesthetized with isoflurane and euthanized via exsanguination. Serum samples were prepared according to the manufacturer's instructions (Sarstedt, cat. No. 41.1500.005). Tissue samples were excised, snap-frozen in liquid nitrogen (unless otherwise indicated), and stored at $-80^{\circ} \mathrm{C}$ until further analysis. All animal procedures were conducted in accordance with institutional guidelines, and ethical approval was obtained from the University Committee on Animal Care (Animal Welfare Assurance no. A3203-01).

\section{Ethanol Feeding}

The chronic ethanol model was used in which 12-wk-old female mice were acclimatized to a liquid diet ad labium without the addition of alcohol for $1 \mathrm{wk}$ then gradually transitioned from $1 \%$ to $5 \%$ ethanol (vol/vol ethanol) over a period of 2 weeks, then maintained at $5 \%$ for the remaining 4 wks. This feeding protocol is believed to reflect chronic ethanol abuse, beginning with low volumes and increasing over time (Breitkopf, et al., 2009) resulting in hepatic steatosis with only moderate inflammation (Bertola, Mathews, Ki, Wang, \& Gao, 2013). On the morning of the final day, food was removed, and mice were fasted for $9 \mathrm{~h}$ before euthanasia. Following 
euthanasia, tissue/serum samples were collected and processed for analysis. The $9 \mathrm{~h}$ time point was selected to be consistent with NIAAA model protocol. As opposed to the previous studies use of control group non-alcohol fed WT mice, the control group was identified as alcohol fed WT mice in order to properly explore the hypothesis of the sex specific benefits of CTRP3. This way, the protective abilities against ALD can be compared between the two alcohol fed cohorts. For this study, eighteen WT mice and ten CTRP3 $\mathrm{Tg}+$ mice were used and all were ethanol fed.

\section{Liver Histology}

As briefly described by (Trogen, et al., 2018) a section of the lateral left lobe of the liver was fixed in $10 \%$ formalin for 2 days. Following fixation, the tissue was dehydrated and embedded in paraffin using standard embedding techniques. Each sample was sectioned at $5 \mu \mathrm{m}$ with a Microm HM325 microtome, mounted, and stained using standard hematoxylin and eosin (H\&E) staining procedures. The tissue was examined and photographed using a Zeiss Axioskop 40 microscope equipped with a Cannon powershot A640 camera.

\section{Immunoblot Analysis}

As briefly described by (Trogen, et al., 2018) liver samples were pulverized in liquid nitrogen, and $\sim 100 \mathrm{mg}$ was suspended in radio immunoprecipitation assay (RIPA) buffer (50mM Tris-HCl, $\mathrm{pH} 8.0,150 \mathrm{mM} \mathrm{NaCl}, .1 \%$ Triton $\mathrm{X}-100, .5 \%$ sodium doxycholate, $.1 \%$ SDS) with protease and phosphatase inhibitors (Bimake, cat. Nos. B14001 and B15001). The samples were incubated for $30 \mathrm{~min}$ at $4^{\circ} \mathrm{C}$ with gentle rotation and then centrifuged at $16,000 \mathrm{~g}$ for $10 \mathrm{~min}$ at $4^{\circ} \mathrm{C}$. The supernatant containing the soluble fraction was collected and prepared for protein quantification. Protein concentrations were determined by commercial assay according to the manufacturer's directions (Thermo, cat. no. 23238). Samples were diluted to equal protein concentrations with RIPA buffer and denatured at $95^{\circ} \mathrm{C}$ for $5 \mathrm{~min}$ after the addition of equal parts 
$2 \times$ SDS loading buffer (4\% SDS, $10 \%$ 2-mercaptoethanol, $20 \%$ glycerol, $0.004 \%$ bromophenal blue, $0.125 \mathrm{M}$ Tris $\cdot \mathrm{HCl}, \mathrm{Ph} 6.8$ ), followed by cooling for $5 \mathrm{~min}$ on ice. Denatured proteins were separated by SDS-polyacrylamide gel electrophoresis, according to the manufacturer's directions (Bio-Rad, cat. no. 456-1046), with the addition of a protein ladder (Bio-Rad, cat. no. 161-0374). After gel electrophoresis, proteins were transferred to a nitrocellulose membrane according to standard procedures (Bio-Rad, cat. no. 1620115). Equal loading was confirmed by Ponceau S stain ( $0.1 \%$ Ponceau S, $5 \%$ acetic acid). Membranes were blocked with $4 \%$ nonfat milk solution for $1 \mathrm{~h}$ at room temperature and then probed with primary antibody (Cell-Signaling Technology cat no. 9102) overnight at $4^{\circ} \mathrm{C}$, followed by appropriate horseradish peroxidase (HRP)conjugated secondary antibody (Thermo Fisher Scientific cat no. 3146). Chemiluminescent signals were detected (chemiluminescent HRP substrate, Millipore, cat. no.

WBKLS0100) with a Bio-rad imager, and band intensities were measured densitometrically and expressed as fold changes relative to those of normal control livers.

\section{Hepatic lipid analysis}

Lipids were extracted as described by Bligh and Dyer and as previously performed $(6,26)$. Briefly, liver samples were weighed and homogenized in phosphate-buffered saline (10 $\mathrm{ml} / \mathrm{g}$ tissue), followed by the addition of 1:2 (vol/vol) chloroform-methanol (3.75 $\mathrm{ml} / \mathrm{ml}$ sample homogenate). Next, chloroform was added (1.2 $\mathrm{ml} / \mathrm{ml}$ sample homogenate), followed by a final addition of distilled water $(1.25 \mathrm{ml} / \mathrm{ml}$ sample homogenate). Samples were vortexed for $30 \mathrm{~s}$ between each step. Samples were then centrifuged $(1,100 \mathrm{~g}$ for $10 \mathrm{~min}$ at room temperature) for phase separation (aqueous phase on top and organic phase below). The lower phase was collected with a glass pipette with gentle positive pressure (so as not to disturb the 
upper phase). Samples were then divided into two aliquots and dried under nitrogen gas at $60^{\circ} \mathrm{C}$. To measure total triglyceride levels, one aliquot from each sample was dissolved in tert-butyl Alcohol-Triton X-100 (3:2 vol/vol) solution. Triglycerides were quantified via colorimetric assay according to the manufacturer's directions (Infinity Triglycerides, Fisher Diagnostics, cat. no. TR22421). 


\section{RESULTS}

\section{Animal Characteristics}

As consistent with past studies utilizing the chronic feeding protocol, there was no statistical difference between the food intake levels of the WT and CTRP3 Tg+ cohorts (Figure 1 A), as well as no difference between body weight, even when normalized in regard to food intake

(Figure 1 B-C). Unexpectedly, there was no statistical difference between the survival proportions in regard to the WT and $\mathrm{Tg}+$ groups. In fact, the $\mathrm{Tg}+$ cohort trended towards a decreased rate of survival in comparison to that of the WT mice ( $78 \%$ in WT versus $44 \%$ in $\mathrm{Tg}, \mathrm{p}=0.13$ ).

A

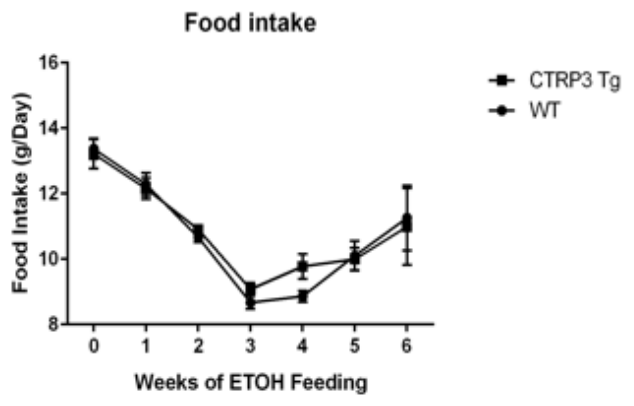

C

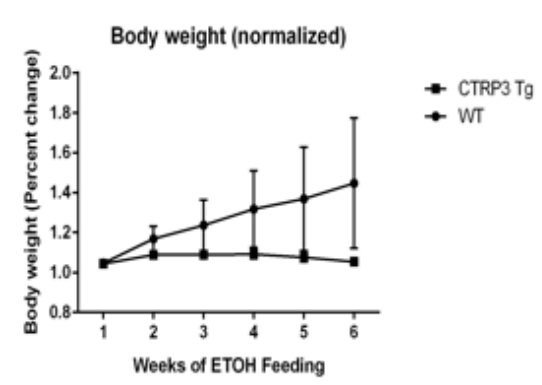

B

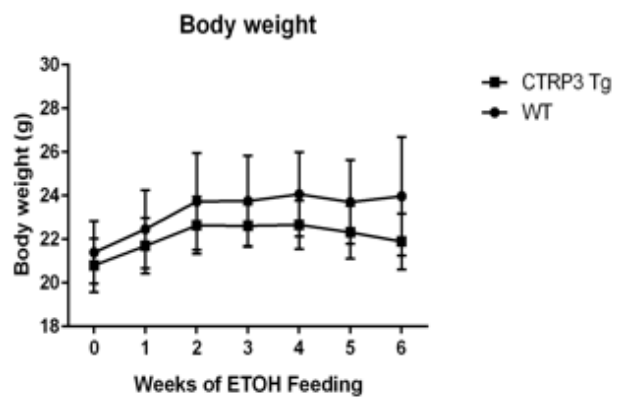

D

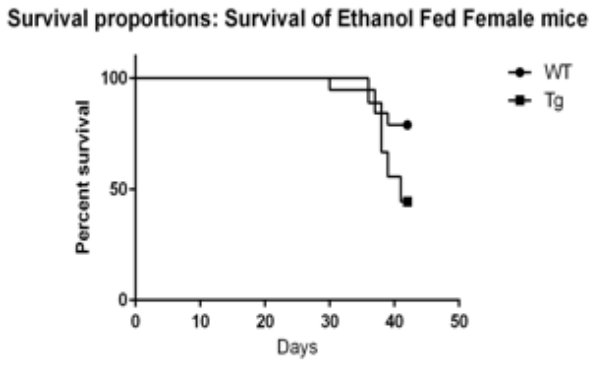

\section{Figure 1. Animal Characteristics}

Transgenic overexpression of C1q tumor necrosis factor-related protein 3 (CTRP3 Tg) in female mice had no effect upon the food intake (A) or body weight (B) when compared to Wildtype (WT) female mice. Daily body weights were also averaged over the week time period for each mouse and normalized to week 0 (C). Between WT and Tg+ female mice in a chronic fed model, there was no significant difference in survival, however, Tg female mice trended $(\mathrm{p}=0.07)$ towards a reduced rate of survival compared to WT female mice (D). Data are reported as means $\pm \mathrm{SD}$. 


\section{Lipid Quantification}

In order to measure the degree of severity of liver steatosis (fatty liver) present in WT vs $\mathrm{Tg}+$ female mice, a qualitative and quantitative analysis of hepatic lipid accumulation was performed. Unexpectedly, a representative qualitative analysis of the WT and $\mathrm{Tg}+$ left lobe liver imaging (figure 2-C), displays no noticeable differences between the hepatic lipid accumulation levels of the two experimental groups. In support of this finding, Stereological analysis (figure 2-A) indicated no difference in the percent of lipid liver volume between the two groups (WT 7.2 \pm 3.6 vs Tg 5.1 $\pm 4.1 \%$ ).

Similarly, figure 2-B indicate no difference in the percent of lipid liver volume between the WT and $\operatorname{Tg}+$ mice.

A

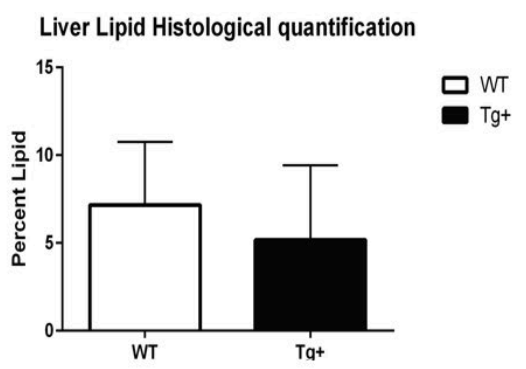

C

B
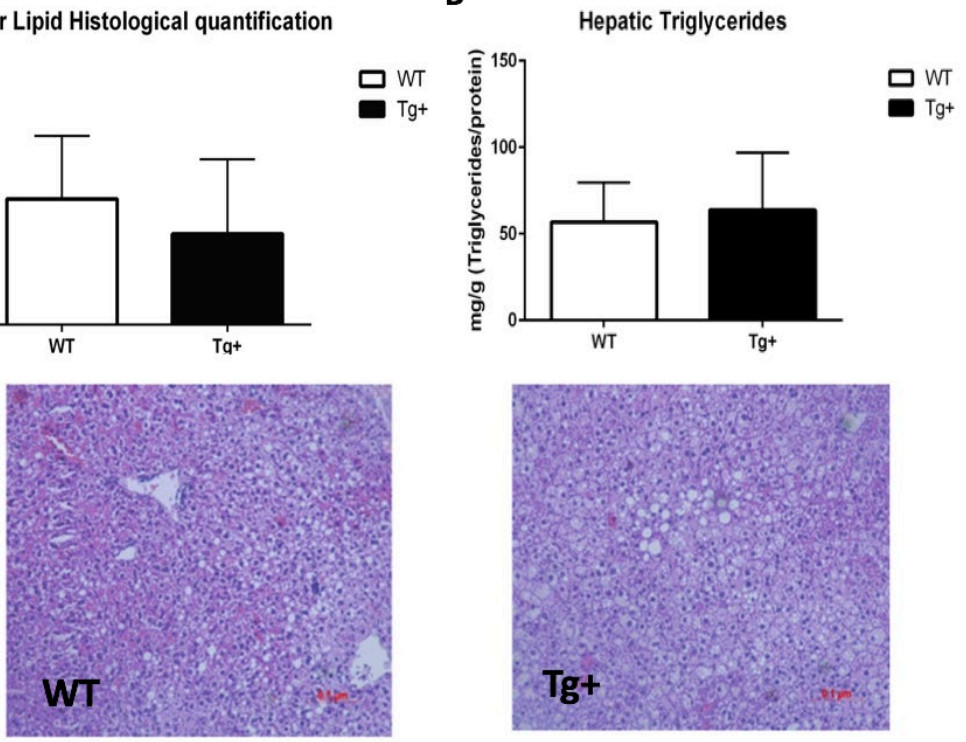

Figure 2. Hepatic lipid content. A) Total hepatic lipid content as a percent area was determined in ethanol fed wild type (WT) and CTRP3 transgenic female mice (Tg+) female mice. B) Total hepatic triglycerides quantification in WT vs Tg+ female mice. C) Representative hematoxylin and eosin-stained images (x20) from left lobe liver sections. Data are reported as means \pm SD. 


\section{Circulating Serum Analytes}

In order to measure the impact of ethanol on inflammation and metabolics, several precursor pro-inflammatory serum analytes concentration levels were examined and compared between the $\mathrm{WT}$ and $\mathrm{Tg}+$ mice. Interestingly, the data in figure 3 displays no statistically significant difference in TNF- $\alpha$ (Figure 1-A) Interleukin 6 (IL-6) (Figure 1-B), Interleukin $1 \beta$ (IL-1 $\beta$ ) (Figure 1-C), Interleukin 10 (IL-10) (Figure 1-D), Interleukin 17 alpha (IL-17alpha) (Figure 1-E), and Interferon gamma (IFN-g) (Figure 1-F) levels. This is indicative of a lack of difference in inflammation between the $\mathrm{WT}, \mathrm{Tg}+$ female mice.

A

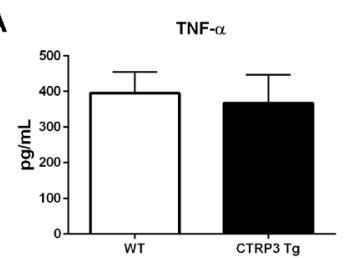

C

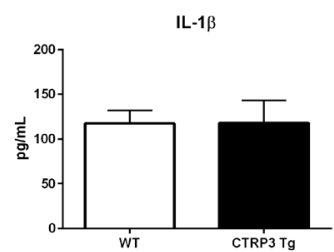

E

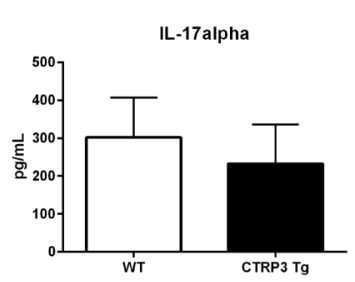

B

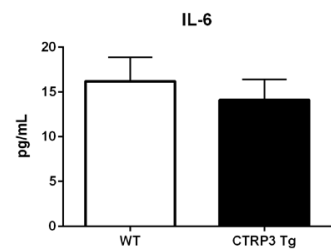

D

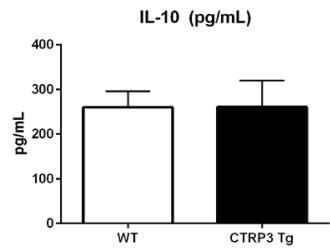

$\mathbf{F}$

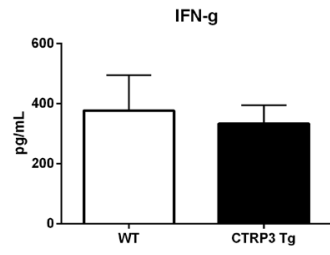

Figure 3. Effects of transgenic overexpression of CTRP3 and chronic ethanol diet on the concentration of circulating serum cytokines.

Concentration of Tumor Necrosis Factor - alpha (TNF- $\alpha$ ) (A), Interleukin 6 (IL-6)(B), Interleukin 1-Beta (IL-1 $\beta$ ), Interleukin 10 (IL-10) (D), Interleukin 17 alpha (IL-17alpha) (E), Interferongamma (IFN-g) (F) in chronic fed Transgenic overexpressed C1q tumor necrosis factor-related protein 3 (CTRP3 Tg) and Wildtype (WT) female mice. Measurements taken in pictograms per milliliter. Data are reported as means \pm SD. 


\section{Circulating ERK 1/2}

The concentration levels of ERK 1/2 (extracellular signal-regulated kinases 1/2) was measured due to its role as a signaling protein of CTRP3, and its proposed role in alcohol induced hepatic damage. Immunoblot assays performed on Bio-Rad Chemidoc technology were utilized to examine optical density and in turn, concentration levels of circulating signaling protein levels. Unexpectedly, as seen in Figure 5, the data displays a slightly higher ERK 1/2 concentration in the $\mathrm{Tg}+$ female mice, which is directly opposite of what was observed in the $\mathrm{Tg}+$ male mice data previously studied. Because of studies involved with the treatment models focusing on blockage of ERK 1/2 resulting in reduced alcohol induced hepatic damage, we would expect to see that if CTRP3 was indeed protective, we would also see reduced ERK $1 / 2$ levels.

\section{ERK 1/2}

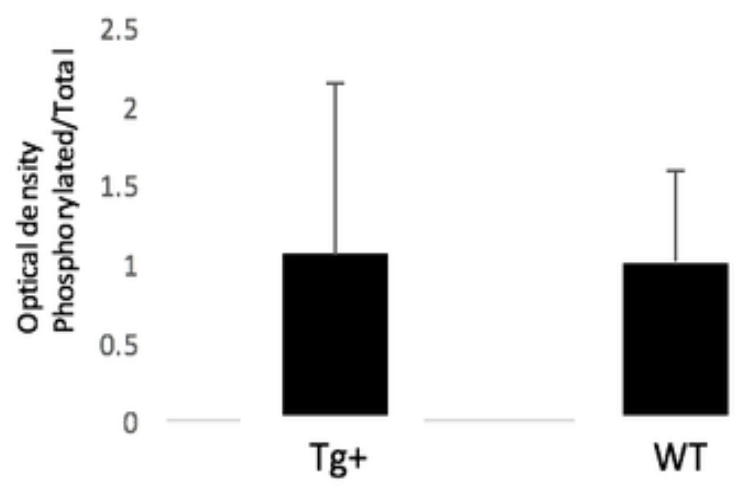

Figure 5. Effect of Transgenic Overexpression of CTRP3 and Chronic Ethanol diet on hepatic phosphorylation of ERK $1 / 2$.

Hepatic ERK $1 / 2$ total and phosphorylated proteins levels were measured in chronic ethanol fed wild type (WT) and CTRP3 transgenic female mice ( $\mathrm{Tg}+$ ). Data reported as optical density of phosphorylated normalized to total protein. Data are reported as means \pm SD. 


\section{DISCUSSION}

The purpose of this study was to determine whether CTRP3 prevented ALD in female mice. Unexpectedly, our findings led us to conclude that CTRP3 does not act protectively in female CTRP $3 \mathrm{Tg}+$ mice, which is in direct contradiction as to what is seen in the previously studied CTRP3 Tg+ male mice. When comparing these two studies, this difference displays the sex-dependent differentiation in the protective mechanism of CTRP3 and ALD. In accordance with past research, we hypothesized a lesser degree of protection in females to account for their historically higher quantity and severity of ALD cases. But the data shows a stark and drastic difference between WT and $\mathrm{Tg}+$ female mice. This can lead to the notion that in females, CTRP3 is acting in a non-protective fashion.

Interestingly, the survival curve displays a non-significant difference between percent survival of WT and CTRP3 Tg+ female mice. Even further, the data trended towards a decreased survival percentage in CTRP3 $\mathrm{Tg}+$ mice. This displays the lack of protection CTRP3 was hypothesized to provide against the progression to chronic stages of alcoholic liver disease. The survival curve is contrary to the previously observed trend in chronic male CTRP3 $\mathrm{Tg}+$ mice, who showed a greater percent survival as compare to WT male chronic fed mice. Similarly, there was no visual differences between the representative hematoxylin and eosin-stained hepatic images taken in both the CTRP3 $\mathrm{Tg}+$ and WT female mice. The similar lipid accumulation levels is further supported by the lack of statistically significant differences in the histological hepatic lipid between the WT and $\mathrm{Tg}+$ female mice. The same trend can be displayed with the lack of statistical significance in hepatic triglyceride levels. Interestingly, this data trended towards a slightly higher triglyceride concentration in the $\mathrm{Tg}+$ female mice as compared to the WT female mice. Since lipid accumulation is indicative of steatosis of the liver, if CTRP 3 were to be protective in female mice as previously seen in the male mice, lipid accumulation levels would 
be lowered in the $\mathrm{Tg}+$ female mice.

The data concerning serum analytes displayed no statistically significant changes in circulating TNF- $\alpha$, IL-6, IL-1B, Il-10, Il-17, and IFN-g. As these analytes are indicative of inflammation, we expected to see a decrease concentration in CTRP3 $\mathrm{Tg}+$ female mice. Lower levels of inflammation are associated with lessened severity of ALD as this is a characteristic of the progression of this disease. In comparison to previous $\mathrm{WT} / \mathrm{Tg}+$ male studies, similar various other pro-inflammatory analytes (insulin, glucagon, resistin, GIP, ghrelin etc..) in the male similarly displayed no statistical difference between the two groups. (Trogen, et al., 2018).

Unexpectedly, ERK1/2 levels were shown to be slightly increased in the female $\mathrm{Tg}+$ mice as opposed to female WT mice. This is significant due to past research involving novel treatments concerning blockage of ERK 1/2. The blockage of ERK 1/2 has been associated with reduced alcohol induced hepatic damage ( $\mathrm{Li}$, et al., 2017). Even though the mechanism of action in this trend is unknown, it is still important to note in relation to CTRP3's protective abilities. If CTRP3 were shown to have be protective in female mice, we would expect the data to trend toward a decrease in ERK $1 / 2$ concentration in the CTRP3 $\mathrm{Tg}+$ levels in females as seen in CTRP3 Tg+ male mice. But instead, the opposite was observed.

Limitations of this study include namely the COVID-19 pandemic that ensued at the end of data collection, largely including the conclusion of immunoblot analysis. As of March $23^{\text {rd }}$, 2020 all institutional courses at East Tennessee State University were moved online and students were not allowed in research facilities. Due to this, immunoblot data including AKT, AMPK, ACC, and others were not able to be concluded and in turn were not able to be included in this manuscript. To expand upon this research, further analysis should be included of various other 
analytes and cytokines to reach further conclusions upon the relationship between the sex specific benefits of CTRP3.

Outside of the detrimental effects of COVID-19 to the research, limitations of the study also include the housing characteristics of this study. The female mice were housed in pairs during the ethanol feeding study (in accordance to protocol) in order to mitigate social stress. Because of this, we are unsure of the specific levels of diet consumption per mouse. As utilized by past studies in the same protocol, the diet consumption is split evenly between the two housed animals to account for food consumption levels.

To further expand upon this study's findings, that CTRP3's protective qualities vary depending on sex, it is imperative to continue to explore these differences. There is a wide array of possible explanations to this disparity, such as sex specific differences in metabolism, hormones, or other processes. Likewise, an interesting direction for further study would be the diagnostic role that CTRP3 can play in ALD. As the early stages of ALD can be nonsymptomatic, looking at how CTRP3 levels can be indicative of ALD severity could be an interesting mechanism to correlate their levels to the progression of the disease. 


\section{CONCLUSION}

The purpose of this study was to determine whether CTRP3 prevented ALD in female mice. However, the data generated did not support the hypothesis. Therefore, we find that overexpression of CTRP3 does not prevent ALD in female mice. In summary, this research displays sex specific qualities of CTRP3 in relation to ALD. CTRP3 protected male mice from ALD, but does not act in a protective manner in female mice. This study has important and far reaching implications as it further builds our knowledge of the sex specific abilities of CTRP3. As most research has primarily used male subjects, the findings of this project highlight the necessity of therapeutic and pharmaceutical studies to report on not only male responses but also on the responses of females. 


\section{REFERENCES}

Acharie, A., \& Jain, S. K. (2017, June). Adiponectin, a Therapeutic Target for Obesity, Diabetes, and Endothelial Dysfunction . International Journal of Molecular Sciences, 16(6), 1321.

Ambient Air Pollution: Health Impacts. (n.d.). (W. H. Organization, Producer) Retrieved Feb 26, 2020, from World Health Organization: https://www.who.int/airpollution/ambient/health-impacts/en/

Bellentani, B., Saccoccio, G., Costa, G., Tiribelli, C., Manenti, F., Sodde, M., . . Brandi, A. (1997, Dec.). Drinking habits as cofactors of risk for alcohol induced liver damage. Gut, 41(6), 845-850.

Bertola, A., Mathews, S., Ki, S. H., Wang, H., \& Gao, B. (2013). Mouse model of chronic and binge ethanol feeding (the NIAAA model) . Natureprotocol, 8, 626-637.

Breitkopf, K., Nagy, L. E., Beier, J. I., Mueller, S., Weng, H., \& Dooley, S. (2009, Jul 23). Current Experimental Perspectives on the Clinical Progression of Alcoholic Liver Disease . Alcohol Clin Exp Res., 33(10), 1647-1655.

DeGroat, A. R., Fleming, C. K., Dunlay, S. M., Hagood, K., Moorman, J. P., \& Peterson, J. M. (2018, November 7). The Sex Specific Effect of Alcohol consumption on circulating levels of CTRP3. (A. Ryabinin, Ed.) PLOS ONE, 13(11), 1-17.

DeGroat, A., Fleming, C., Dunlay, S., Hagood, K., Moorman, J., \& Peterson, J. (2018, Nov 7). The sex specific effect of alcohol consumptuion on circulating levels of CTRP3. (A. E. Ryabinin, Ed.) PLOS one, 13(11), 1-17.

Deng, Y., \& Scherer, P. E. (2010, Nov). Adipokines as novel biomarkers and regulators of the metabolic syndrome. ANNALS fo the New York Academy of Sciences, E1-D9.

Drinking Levels Defined. (n.d.). Retrieved from National Institue on Alcohol Abuse and Alcoholism : https://www.niaaa.nih.gov/alcohol-health/overview-alcoholconsumption/moderate-binge-drinking

Fields, R. D. (2014, June 18). NIH policy: Mandate goes too far. nature (340), 510.

Gamberi, T., Maherinie, F., Modesti, A., \& Fiashi, T. (2018, May 7). Adiponectin Signaling Pathways in Liver Diseases. Biomedicines, 6(2).

Gao, B., \& Bataller, R. (2011, Nov). Alcoholic Liver Disease: Pathogenesis and New Therapeutic Targets. Gastroenterology, 141(5), 1572-1585.

Han, S., Kim, J., Lee, S., Jeong, A. L., Park, J. S., Yong, H. J., .. Yang, Y. (2016, May 23). Circulating CTRP1 Levels in Type 2 Diabetes and Their Association with FGF21 . International Journal of Endocrinology, 2016.

Kema, V. H., Mojerla, N. R., Khan, I., \& Mandal, P. (2015, Apr 2). Effect of alcohol on adipose tissue: a review on ethanol mediated adipose tissue injury. Adipocyte, 4(4), 225-231.

Knell, A. J. (1980, July). Liver Function and Failure. Journal of the Royal College of Physicians of London, 14(3), 205-208.

Li, C., Li, L., Yang, C., Zhong, Y., Wu, D., Shi, L., \& L, C. (2017, Nov 4). Hepatoprotective effects of Methyl ferulic acid on alcohol-induced liver oxidative injury in mice by inhibiting the NOX4/ROS-MAPK pathway. . Biochem Biophys Res Commun., 493(1), 277-285.

Li, Y., Wright, G. L., \& Peterson, J. M. (2017, June 18). C1q/TNF-Related Protein 3 (CTRP3) Function and Regulation. Comprehensive Physiology , 7(3), 863-878. 
Lieber, C. S. (2004, August ). Alcoholic fatty liver: its pathogenesis and mechanism of progression to inflammation and fibrosis . Alcohol, 34(1), 9-19.

Lundbom, J. (2017, Jul 6). Adipose tissue and Liver. Journal of Applied Physiology , 162-167.

Maher, J. J. (1997). Exploring Alcohol's Effects on Liver Funciton. Alcohol Health \& Research World, 21(1), 5-12.

Mazure, C. M., \& Jones, D. P. (2015, Oct 26). Twenty years and still counting: including women as participants and studying sex and gender in biomedical research . BMC Women's health, 15(94).

Mumenthaler, M. S., Taylor, J. L., O'Hara, R., \& Yesavage, J. A. (1999). Gender Differences in Moderate Drinking Effects . Alcohol Research Current Reviews, 23(1), 55-64.

Osna, N. A., Donohue, T. M., \& Kharbanda, K. K. (2017). Alcoholic Liver Disease: Pathogenesis and Current Management. Alcohol Resarch Current Reviews, 38(2), 146-161.

Pereira, S., \& Alvarez-Leite , J. (2013, May 15). Adipokines: biological funcions and metabolically healthy obese profile. Journal of Receptor, Ligand and Channel Research, 7, 15-25.

Peterson, J. M., Aja, S., Wei, Z., \& Wong, W. G. (2011, Nov 15). CTRP1 Protein Enhances Fatty Acid Oxidation via AMP-activated Protein Kinase (AMPK) Activation and Acetyl-CoA Carboxylase (ACC) Inhibition* . Journal of Biological Chemistry, 287(2), 1576-1587.

Peterson, J. M., Seldin, M. W., \& Wong, G. W. (2013, Jun 5). CTRP3 attenuates diet-induced hepatic steatosis by regulating triglyceride metabolism. . American journal of physiology - Gastrointestinal and liver physiology, G214-G224.

Rodriguez, S., Lie, X., Petersen, P., Tan, S., Little, H. c., \& Wong, G. W. (2016, Oct 1). Loss of CTRP1 disrupts glucose and lipid homeostasis . American Physiological Society, 311(4), E678-E697.

Sakhuja, P. (2014, Nov 28). Pathology of alcoholic liver disease, can it be differentiated from nonalcoholic steatohepatitis? . World Journal of Gastroenterology , 20(44), 1647416479.

Tang, H., Sebastian, B. M., Axhemi, A., Chen, X., Hillian, A. D., Jacobsen, D. W., \& Nagy, L. E. (2012, February). Ethanol-induced oxidative stress via the CYP2E1 pathway disrupts adiponectin secretion from adipocytes . Alcohol Clin Exp Res., 36(2), 214-222.

Trogen, G., Alamian, A., \& Peterson, J. M. (2019, Nov 9). High molecular weight, but not total, CTRP3 levels are associated with serum triglyceride levels. Physiological Reports.

Trogen, G., Bacon, J., Li, Y., Wright, G., Degroat, A., Hagood, K., . . Peterson, J. M. (2018, May 15). Transgenic overexpression of CTRP3 prevents alcohol-induced hepatic triglyceride accumulation. Am J Physiol Endocrinol Metab, E949-E960. 